\title{
Correlation and path coefficient analysis of body weight and morphometric traits of two exotic genetic groups of ducks in Nigeria
}

\author{
A Yakubu* ${ }^{1}$, MM Muhammed $^{2}$, MM Ari $^{1}$, IS Musa-Azara ${ }^{2}$, J N Omeje \\ ${ }^{1}$ Department of Animal Science, Faculty of Agriculture, Nasarawa State University, Keffi, Shabu-Lafia Campus, \\ PMB 135 Lafia, Nasarawa State; ${ }^{2}$ Department of Animal Science, College of Agriculture, Lafia, Nasarawa State; \\ ${ }^{3}$ Department of Veterinary Medicine and Surgery, University of Abuja, Nigeria
}

\begin{abstract}
The study aimed at describing objectively the interdependence between body weight (BWT) and morphometric traits in Khaki Campbell and Pekin ducks using multivariate path analysis technique. Measurements were taken on one hundred and ninety seven (197) randomly selected 10-week old Khaki Campbell and Pekin ducks, respectively in Plateau State, Nigeria. The birds were reared on deep litter in a semi-intensive system where they were kept in a fenced area provided with water ponds and locked up in the poultry house during the night. The body parts measured were, body length (BDL); thigh length (THL); thigh circumference (THC); breast circumference (BTC); bill length (BLL); neck length $(N K L)$; neck circumference (NKC); shank length (SHL); shank width (SHW); total leg length (TLL) and wing length (WL). General linear model was used to study genotype and sex effects. Pekin ducks had a superior advantage $(p<0.05)$ over their Khaki Campbell counterparts in all the body parameters estimated. Sexual dimorphism $(p<0.05)$ was in favour of male ducks. Pairwise phenotypic correlations between BWT and morphometric traits were positive and significant $(p<0.01)$, ranging from $0.38-0.95$ and 0.35-0.92 for Khaki Campbell and Pekin ducks, respectively. Path analysis revealed that BDL was the variable of utmost importance directly influencing BWT in male Khaki Campbell and Pekin ducks (path coefficient $=0.535$ and 0.508 , respectively; $p<0.01$ ) while BTC and SHL were the most responsible parameters affecting BWT in female Khaki Campbell and Pekin ducks [path coefficient $=0.594(P<0.01)$ and $1.197(p<0.05)$, respectively]. The optimum regression models for the prediction of BWT in Khaki Campbell ducks included BDL, SHL, BTC and NKC (male) and BDL, WNL and BTC (female); while in their Pekin counterparts, BDL, BLL and BTC (male) and BDL and SHL (female) were incorporated.
\end{abstract}

Key words: Exotic ducks, morphometric traits, multivariate analysis, phenotypic correlation

Bangladesh Animal Husbandry Association. All rights reserved.

Bang. J. Anim. Sci. 2015. 44 (1): 1-9

\section{Introduction}

Waterfowl production has been on an upward trend and has become increasingly important around the world over time. The production of duck has increased during the last 100 years and was six-fold over 1961 with world duck meat production reaching 3,980,136 ton (Huang et al. 2010). Ducks have better adaptation to various environmental conditions compared to chickens. They are hardy and can withstand a number of diseases. They can also scavenge on their own and require less man power to keep (Adzitey and Adzitey 2011). Pekin and Khaki Campbell are among the exotic ducks found in Nigeria. The true Pekin originated in China and is now widespread throughout the world. White Pekin duck perform better than the modern broiler chicken in terms of weight gain and feed efficiency due to genetic improvement. The Khaki Campbell on the other hand originated in England by crossing Rouen, White Indian Runner and Wild Mallards. Drakes weight about $3 \mathrm{~kg}$ while ducks are about $2.5 \mathrm{~kg}$ live weight. To counteract the continuing genetic erosion of the poultry population, preventive measures are needed to create conservative and reserve poultry flocks differing in origin, conformation and productivity (Kokoszyński and Bernacki 2011). Maintenance of conservation of poultry flocks of different origin and genotype seems necessary for biological, economic, cultural and historical reasons. In 


\section{Correlation and path coefficient analysis on traits of ducks}

order to adopt strategies of conservation and improvement, it is necessary to estimate the amount and distribution of genetic variation in preselected populations of duck genetic resources.

Information on morphological characteristics is a prerequisite to sustainable breed improvement, utilization and conservation. Body size and shape measured objectively could improve selection for growth by enabling the breeder to recognize early maturing and late maturing animals of different sizes. Based on body conformation, meat production can better be estimated than other production properties making body measurements important selection criteria. However, the biological relationships among the morphometric traits may be different if these body measurements are treated as bivariates rather than multi-variates (Eyduran et al. 2010; Mir et al. 2014). This is because simple correlation only allows an assessment of the magnitude and direction of the association between the two characters, without providing the necessary information relating to direct and indirect effects of a group of characters in relation to a dependent variable of the greatest importance (Lorentz et al. 2011). In order to obviate this deficiency, the use of a multivariate technique, path analysis may be more appropriate. Path analysis is a device that a breeding researcher can use to break the correlation of direct and indirect effects through basic variables such as body yields, and explanatory variables such as morphometric measures, thereby providing a better understanding of the reasons for the associations between these traits (Neto et al. 2012).

While efforts have been made to phenotypically characterize the indigenous Muscovy ducks of Nigeria (Yakubu 2011, 2013), there is dearth of information on the body weight and morphometric traits of exotic ducks found in the country. The present study therefore aimed at evaluating, through path analysis of phenotypic correlations, the morphometric variables that are more directly related to the body weight of Khaki Campbell and Pekin ducks, respectively under the humid tropical environment of Nigeria, subsaharan Africa.

\section{Materials and Methods}

The study was carried out at the Duck Unit of the National Veterinary Research Institute, Vom-Jos, Plateau State, north-central Nigeria from MarchDecember, 2012. It is found on Latitude $09^{\circ} 44^{\prime}$ $\mathrm{N}$ and Longitude $08^{\circ} 45^{\prime} \mathrm{E}$. The Jos Plateau comprises an area of $8000 \mathrm{~km}^{2}$ with an average altitude of $1280 \mathrm{~m}$. The annual rainfall (1000 $1500 \mathrm{~mm}$ ) is between May and October while the dry season runs from November to April. The birds were reared on deep litter in a semiintensive system where they were kept in a fenced area during the day, provided with water ponds and locked up in the poultry house during the night. Birds were fed grower ration while clean potable water was freely available. Routine vaccination and other management practices were done. Some of the management practices included, cleaning the feeders and water containers daily; addition of fresh feed to the stale feed in the feeders after the litter and droppings in the latter have been removed; periodic turning of the feeders to ensure feeding to appetite and changing of caked litter periodically. The foot bath was also changed regularly. Dead birds were removed promptly to prevent the contamination of the other birds which might peck them.

Data were obtained from a total of one hundred and ninety seven (197) randomly selected, 10week old Khaki Campbell (45 males and 45 females), and Pekin (62 males and 45 females) ducks, respectively. Body weight (BWT), body length (BDL), thigh length ( $T H L)$, thigh circumference (THC), breast circumference (BTC), bill length (BLL), neck length (NKL), neck circumference $(N K C)$, shank length $(S H L)$, shank width (SHW), total leg length (TLL) and wing length (WNL). The anatomical reference points were as earlier described (Teguia et al. 2008, Yakubu 2011). The descriptive statistics of body weight and morphometric measurements were computed. The ANOVA was used to assess genetic group effect on body weight and morphometric measurements of the two duck breeds. Sex effect, irrespective of genetic group, was also assessed. The employed linear model was: 
$\mathbf{Y}_{\mathrm{ijk}}=\boldsymbol{\mu}+\boldsymbol{G}_{\mathrm{i}}+S_{\mathrm{j}}+\mathbf{e}_{\mathrm{ijk}}$

where, $Y_{i j k}=$ individual observation; $\mu=$ overall mean

$G_{i}=$ fixed effect of ith genotype ( $i=$ Khaki Campbell, Pekin); $S_{j}=$ fixed effect of $k^{\text {th }}$ sex ( $k=$ male, female); $e_{i j k}=$ random error associated with each record (normally, independently and identically distributed with zero mean and constant variance)

Pair-wise correlations among body weight and morphometric characters of each sex in each genetic group were also determined. In order to obtain a preliminary predictive model, the multiple linear regression equation was adopted as follows:

$\mathbf{Y}=\mathbf{a}+\mathbf{b}_{1} \mathbf{X}_{1}+\mathbf{b}_{2} \mathbf{X}_{2}+\mathbf{b}_{3} \mathbf{X}_{3}+\ldots \ldots \ldots+\mathbf{b}_{\mathrm{p}} \mathbf{X}_{\mathrm{p}}$

where, $Y=$ dependent or endogenous variable (BWT); $a$ = intercept; $b$ 's = regression coefficients; $X$ 's = independent or exogenous variables (BDL, THL, THC, BTC, BLL, NKL, NC, SHL, SHW, TLL, $W L)$.

Standardized partial regression coefficients called path coefficients (beta weights) were calculated. This was to allow direct comparison of values to reflect the relative importance of independent variables to explain variation in the dependent variable. The path coefficient from an explanatory variable $(X)$ to a response variable $(Y)$ was as described by Mendes et al. (2005). The significance of each path coefficient in the multiple linear regression model was tested by tstatistic.

The indirect effects of $X_{i}$ on $Y$ through $X_{j}$ were calculated as follows:

$I_{Y X i}=r_{X i X j} P_{Y . X j}$

where, $I E_{Y X_{i}}=$ the direct effect of $X_{i}$ via $X_{j}$ on $Y ; r_{X_{i X j}}=$ correlation coefficient between ith and jth independent variables; $P_{Y . X j}=$ path coefficient that indicates the direct effect of jth independent (exogenous) variable on the dependent (endogenous) variable

All the statistical analyses were done using SPSS (2010).

\section{Results}

The means $( \pm \mathrm{SE})$ and coefficients of variation of BWT and biometric traits of the two genetic groups and sexes investigated are presented in Table 1. Genotype had significant $(P<0.05)$ effect on all the body parameters with higher values recorded for Pekin ducks compared to their Khaki Campbell counterparts. Sexual dimorphism was also observed in all the traits with higher values $(p<0.05)$ recorded for male ducks compared to the females.

Table 1. Means, standard errors (SE) and coefficients of variation (CV, \%) for body weight ( $\mathrm{kg}$ ) and morphometric traits $(\mathrm{cm})$ of Khaki Campbell and Pekin according to genotype and sex

\begin{tabular}{lcccccccc}
\hline \multirow{2}{*}{ Trait } & \multicolumn{2}{c}{ Khaki Campbell } & \multicolumn{2}{c}{ Pekin } & \multicolumn{2}{c}{ Male } & \multicolumn{2}{c}{ Female } \\
\cline { 2 - 9 } & Mean \pm SE & CV & Mean \pm SE & CV & Mean $\pm S E$ & CV & Mean $\pm S E$ & CV \\
\hline BWT & $1.49 \pm 0.01^{\mathrm{b}}$ & 8.72 & $1.55 \pm 0.01^{\mathrm{a}}$ & 9.03 & $1.60 \pm 0.01^{\mathrm{a}}$ & 6.88 & $1.43 \pm 0.01^{\mathrm{b}}$ & 6.99 \\
BDL & $44.95 \pm 0.33^{\mathrm{b}}$ & 6.96 & $46.94 \pm 0.36^{\mathrm{a}}$ & 7.95 & $48.14 \pm 0.29^{\mathrm{a}}$ & 6.21 & $43.52 \pm 0.26^{\mathrm{b}}$ & 5.72 \\
BLL & $4.44 \pm 0.04^{\mathrm{b}}$ & 9.03 & $4.69 \pm 0.05^{\mathrm{a}}$ & 11.09 & $4.80 \pm 0.04^{\mathrm{a}}$ & 8.96 & $4.30 \pm 0.04^{\mathrm{b}}$ & 9.53 \\
BTC & $31.51 \pm 0.24^{\mathrm{b}}$ & 7.33 & $33.00 \pm 0.20^{\mathrm{a}}$ & 6.30 & $33.51 \pm 0.17^{\mathrm{a}}$ & 5.31 & $30.92 \pm 0.22^{\mathrm{b}}$ & 6.63 \\
NKC & $8.38 \pm 0.05^{\mathrm{b}}$ & 5.25 & $8.77 \pm 0.06^{\mathrm{a}}$ & 7.41 & $8.85 \pm 0.06^{\mathrm{a}}$ & 7.12 & $8.27 \pm 0.04^{\mathrm{b}}$ & 4.23 \\
NKL & $13.24 \pm 0.16^{\mathrm{b}}$ & 11.40 & $14.07 \pm 0.16^{\mathrm{a}}$ & 11.73 & $14.38 \pm 0.16^{\mathrm{a}}$ & 11.40 & $12.87 \pm 0.13^{\mathrm{b}}$ & 9.40 \\
SHL & $4.06 \pm 0.04^{\mathrm{b}}$ & 9.61 & $4.51 \pm 0.08^{\mathrm{a}}$ & 19.29 & $4.59 \pm 0.07^{\mathrm{a}}$ & 16.77 & $3.96 \pm 0.05^{\mathrm{b}}$ & 12.12 \\
SHW & $3.85 \pm 0.03^{\mathrm{b}}$ & 7.79 & $4.14 \pm 0.05^{\mathrm{a}}$ & 13.04 & $4.20 \pm 0.05^{\mathrm{a}}$ & 11.19 & $3.78 \pm 0.04^{\mathrm{b}}$ & 9.26 \\
THL & $7.79 \pm 0.08^{\mathrm{b}}$ & 9.88 & $8.26 \pm 0.10^{\mathrm{a}}$ & 11.99 & $8.54 \pm 0.08^{\mathrm{a}}$ & 9.72 & $7.45 \pm 0.07^{\mathrm{b}}$ & 8.46 \\
THC & $7.65 \pm 0.05^{\mathrm{b}}$ & 6.41 & $7.94 \pm 0.06^{\mathrm{a}}$ & 8.19 & $8.15 \pm 0.05^{\mathrm{a}}$ & 6.38 & $7.39 \pm 0.04^{\mathrm{b}}$ & 5.14 \\
TLL & $11.80 \pm 0.12^{\mathrm{b}}$ & 9.49 & $12.76 \pm 0.14^{\mathrm{a}}$ & 11.60 & $13.03 \pm 0.13^{\mathrm{a}}$ & 10.51 & $11.41 \pm 0.10^{\mathrm{b}}$ & 7.93 \\
WNL & $21.34 \pm 0.30^{\mathrm{b}}$ & 13.17 & $23.13 \pm 0.27^{\mathrm{a}}$ & 12.19 & $24.06 \pm 0.21^{\mathrm{a}}$ & 9.10 & $20.23 \pm 0.24^{\mathrm{b}}$ & 11.02 \\
\hline
\end{tabular}

BWT, body weight; BDL, body length; BLL, bill length; BTC, breast circumference; NKC, neck circumference; NKL, neck length; SHL, shank length; SHW, shank width; THL, thigh length; THC, thigh circumference; TLL, total leg length; WNL, wing length; Means within the same row with different superscripts differed significantly $(p<0.05)$ for genotype and sex effects, respectively 
All phenotypic correlation coefficients between BWT and other body measurements in the two exotic ducks were positive and significant. In Khaki Campbells, the coefficients ranged from 0.47-0.95 and 0.38-0.92 for drakes and ducks, respectively (Table 2). In their Pekin counterparts, correlation coefficients (0.61-0.90, drakes) and (0.35-0.92, ducks) were recorded (Table 3 ). The correlations among the rest of the body measurements ranged from low to high values in both genetic groups.

Preliminary regression equations from simple phenotypic correlations among BWT and the 11 morphometric traits of Khaki Campbell and Pekin ducks investigated in this study are shown in Table 4.

Table 2. Phenotypic correlations for body weight and morphometric traits of Khaki Campbell ducks based on sex**

\begin{tabular}{lllllllllllll}
\hline Traits & BWT & BDL & NKL & BLL & WNL & SHL & SHW & THL & THC & BTC & TLL & NKC \\
\hline BWT & & 0.95 & 0.74 & 0.77 & 0.74 & 0.60 & 0.47 & 0.85 & 0.85 & 0.90 & 0.90 & 0.51 \\
BDL & 0.91 & & 0.62 & 0.76 & 0.75 & 0.48 & 0.40 & 0.85 & 0.83 & 0.88 & 0.87 & 0.42 \\
NKL & 0.69 & 0.69 & & 0.62 & 0.56 & 0.57 & 0.43 & 0.72 & 0.74 & 0.66 & 0.77 & 0.53 \\
BLL & 0.38 & $0.25^{\text {ns }}$ & $0.27^{\text {ns }}$ & & 0.84 & 0.47 & $0.34^{*}$ & 0.57 & 0.73 & 0.76 & 0.61 & $0.18^{\text {ns }}$ \\
WNL & 0.79 & 0.63 & 0.59 & 0.65 & & 0.45 & $0.27^{\text {ns }}$ & 0.74 & 0.79 & 0.60 & 0.71 & $0.30^{*}$ \\
SHL & 0.63 & 0.70 & 0.39 & $0.20^{\text {ns }}$ & 0.44 & & 0.55 & 0.45 & 0.61 & 0.42 & 0.58 & $0.37^{\star}$ \\
SHW & 0.92 & 0.89 & 0.72 & $0.36^{*}$ & 0.71 & 0.65 & & $0.32^{*}$ & 0.38 & 0.37 & 0.37 & -0.09 \\
THL & 0.53 & 0.73 & 0.49 & $0.16^{\text {ns }}$ & $0.13^{\text {ns }}$ & 0.43 & 0.59 & & 0.85 & 0.71 & 0.97 & 0.60 \\
THC & 0.64 & 0.43 & $0.34^{*}$ & 0.53 & 0.80 & $0.35^{\star}$ & 0.60 & $-0.07^{\text {ns }}$ & & 0.71 & 0.89 & 0.58 \\
BTC & 0.90 & 0.82 & 0.59 & $0.34^{*}$ & 0.66 & 0.74 & 0.92 & 0.44 & 0.60 & & 0.76 & 0.38 \\
TLL & 0.49 & 0.62 & 0.52 & $0.04^{\text {ns }}$ & $0.13^{\text {ns }}$ & 0.40 & 0.59 & 0.74 & $0.03^{\text {ns }}$ & 0.45 & & 0.68 \\
NKC & 0.45 & $0.35^{\text {ns }}$ & $0.21^{\text {ns }}$ & $0.29^{\text {ns }}$ & 0.45 & $0.37^{*}$ & 0.46 & $0.13^{\text {ns }}$ & 0.48 & 0.42 & $0.09^{\text {ns }}$ & \\
\hline
\end{tabular}

${ }^{*}$, significant at $p<0.01$ for all correlation coefficients except otherwise stated; ${ }^{*}$, significant at $\left.p<0.05\right), n s$, not significant; Upper diagonal were male; Lower diagonal were female

Table 3. Phenotypic correlations for body weight and morphometric traits of Pekin ducks based on sex**

\begin{tabular}{lllllllllllll}
\hline Traits & BWT & BDL & NKL & BLL & WNL & SHL & SHW & THL & THC & BTC & TLL & NKC \\
\hline BWT & & 0.90 & 0.61 & 0.62 & 0.75 & 0.74 & 0.73 & 0.82 & 0.81 & 0.86 & 0.78 & 0.64 \\
BDL & 0.92 & & 0.47 & 0.34 & 0.71 & 0.59 & 0.60 & 0.73 & 0.67 & 0.86 & 0.71 & 0.56 \\
NKL & 0.89 & 0.91 & & 0.59 & 0.67 & 0.77 & 0.76 & 0.76 & 0.81 & 0.54 & 0.77 & 0.69 \\
BLL & 0.53 & 0.44 & 0.50 & & 0.61 & 0.67 & 0.64 & 0.66 & 0.79 & 0.33 & 0.58 & 0.50 \\
WNL & $0.35^{\star}$ & $0.23^{\text {ns }}$ & 0.44 & 0.44 & & 0.70 & 0.70 & 0.73 & 0.80 & 0.64 & 0.80 & 0.52 \\
SHL & 0.88 & 0.81 & 0.89 & 0.74 & 0.50 & & 0.96 & 0.85 & 0.94 & 0.56 & 0.87 & 0.84 \\
SHW & 0.89 & 0.85 & 0.95 & 0.68 & 0.51 & 0.97 & & 0.82 & 0.91 & 0.54 & 0.87 & 0.75 \\
THL & 0.84 & 0.84 & 0.92 & 0.47 & 0.51 & 0.90 & 0.91 & & 0.92 & 0.71 & 0.91 & 0.82 \\
THC & 0.80 & 0.71 & 0.85 & 0.75 & 0.72 & 0.91 & 0.93 & 0.79 & & 0.61 & 0.92 & 0.83 \\
BTC & 0.83 & 0.85 & 0.85 & 0.54 & $0.11^{\text {ns }}$ & 0.78 & 0.83 & 0.67 & 0.72 & & 0.68 & 0.56 \\
TLL & 0.79 & 0.79 & 0.79 & 0.58 & 0.19 ns & 0.82 & 0.79 & 0.80 & 0.65 & 0.78 & & 0.80 \\
NKC & 0.75 & 0.65 & 0.72 & 0.64 & 0.41 & 0.87 & 0.83 & 0.72 & 0.85 & 0.68 & 0.65 & \\
\hline
\end{tabular}

${ }^{* *}$, significant at $p<0.01$ for all correlation coefficients except otherwise stated; ${ }^{*}$, significant at $\left.p<0.05\right), n s$, not significant; Upper diagonal were male; Lower diagonal were female 
Path coefficients of the explanatory variables of the sexes of the two exotic genetic groups are presented in Tables 5, 6, 7 and 8. BDL had the greatest direct influence on BWT in male Khaki Campbell and Pekin ducks (path coefficient= 0.535 and 0.508 , respectively; $p<0.01$ ). Although BTC significantly affected BWT in male Pekins, it was greatly indirectly influenced by BDL. However, BTC and SHL were the most responsible parameters directly affecting BWT in female Khaki Campbell and Pekin ducks [path coefficient $=0.594(p<0.01)$ and $1.197(p<0.05)$, respectively]. Although NKL, THL and THC had high and simple significant correlations with BWT, their direct effects were non-significant in both Khaki Campbell and Pekin ducks, respectively as they were considerably realized via BDL.
The path coefficients of NKL, BLL, WNL, SHW, THL, THC and TLL (males) and NKL, BLL, SHW, THL, THC, TLL, NKC (females) in Khaki Campbell ducks were statistically non-significant, as revealed by the t-test. This is an indication that these variables were less significant in the estimation of BWT, as they were realized considerably via BDL. Although the effect of SHL in female Khaki Campbells was significant, it was negatively impacted. All these variables were thus expunged from the optimum regression models to obtain much more simplified equations. Similarly, NKL, WNL, SHL, SHW, THL, THC, TLL and NKC (males) and NKL, BLL, WNL, SHW, THL, THC, BTC, TLL and NKC (females) in Pekins did not have direct significant effects on BWT, and were thus deleted from the final regression equations.

Table 4. Preliminary regression models for the estimation of body weight from morphometric traits of Khaki Campbell and Pekin ducks

\begin{tabular}{|c|c|c|c|}
\hline Breed & Model & S.E. & $\mathrm{R}^{2}$ \\
\hline \multicolumn{4}{|c|}{ Khaki Campbell } \\
\hline Male & $\begin{aligned} \mathrm{BTW}= & -0.648+0.023 \mathrm{BDL}+0.006 \mathrm{NKL}+0.020 \mathrm{BLL}+0.003 \mathrm{WNL}+0.037 \mathrm{SHL}+0.037 \mathrm{SHW}+0.028 \mathrm{THL}-0.026 \mathrm{THC}+0.015 \mathrm{BTC} \\
& -0.021 \mathrm{TLL}+0.041 \mathrm{NKC}\end{aligned}$ & 0.02 & 0.97 \\
\hline Female & $\begin{aligned} \mathrm{BTW}= & -0.336+0.023 \mathrm{BDL}+0.002 \mathrm{NKL}-0.012 \mathrm{BLL}+0.013 \mathrm{WNL}-0.082 \mathrm{SHL}-0.097 \mathrm{SHW}+0.000 \mathrm{THL}+0.014 \mathrm{THC}+0.027 \mathrm{BTC} \\
& +0.011 \mathrm{TLL}+0.023 \mathrm{NKC}\end{aligned}$ & 0.02 & 0.96 \\
\hline \multicolumn{4}{|l|}{ Pekin } \\
\hline Male & $\begin{aligned} \mathrm{BTW}= & -0.376+0.017 \mathrm{BDL}-0.002 \mathrm{NKL}+0.058 \mathrm{BLL}-0.004 \mathrm{WNL}+0.011 \mathrm{SHL}+0.008 \mathrm{SHW}-0.003 \mathrm{THL}+0.031 \mathrm{THC}+0.025 \mathrm{BTC} \\
& -0.003 \mathrm{TLL}-0.011 \mathrm{NKC}\end{aligned}$ & 0.02 & 0.95 \\
\hline Female & $\begin{aligned} \mathrm{BTW}= & 0.320+0.029 \mathrm{BDL}+0.020 \mathrm{NKL}-0.068 \mathrm{BLL}+0.010 \mathrm{WNL}+0.214 \mathrm{SHL}-0.076 \mathrm{SHW}-0.095 \mathrm{THL}-0.045 \mathrm{THC}-0.002 \mathrm{BTC} \\
& +0.008 \mathrm{TLL}+0.009 \mathrm{NKC}\end{aligned}$ & 0.04 & 0.92 \\
\hline
\end{tabular}

$S E$, standard error; $R^{2}$, coefficient of determination

Table 5. Direct and Indirect effect of morphometric traits on body weight of male Khaki Campbell ducks

\begin{tabular}{|c|c|c|c|c|c|c|c|c|c|c|c|c|}
\hline Trait & DE & Indirec & fect & & & & & & & & & \\
\hline & & BDL & NKL & BLL & WNL & SHL & SHW & THL & THC & BTC & TLL & NKC \\
\hline $\mathrm{BDL}$ & $0.535^{\star \star}$ & & 0.052 & 0.043 & 0.042 & 0.064 & 0.036 & 0.150 & -0.088 & 0.215 & -0.174 & 0.075 \\
\hline NKL & $0.084^{\text {ns }}$ & 0.332 & & 0.035 & 0.031 & 0.076 & 0.038 & 0.127 & -0.079 & 0.161 & -0.154 & 0.095 \\
\hline BLL & 0.056 ns & 0.407 & 0.052 & & 0.047 & 0.063 & 0.030 & 0.101 & -0.078 & 0.185 & -0.122 & 0.032 \\
\hline WNL & $0.056^{\text {ns }}$ & 0.401 & 0.047 & 0.047 & & 0.060 & 0.024 & 0.131 & -0.085 & 0.146 & -0.142 & 0.054 \\
\hline SHL & $0.133^{\star}$ & 0.257 & 0.048 & 0.026 & 0.025 & & 0.049 & 0.080 & -0.065 & 0.102 & -0.116 & 0.066 \\
\hline SHW & 0.089 ns & 0.214 & 0.036 & 0.019 & 0.015 & 0.073 & & 0.057 & -0.041 & 0.090 & -0.074 & 0.016 \\
\hline THL & $0.177 \mathrm{~ns}$ & 0.455 & 0.060 & 0.032 & 0.041 & 0.060 & 0.028 & & -0.091 & 0.173 & -0.194 & 0.107 \\
\hline THC & $-0.107^{\mathrm{ns}}$ & 0.444 & 0.062 & 0.041 & 0.044 & 0.081 & 0.034 & 0.150 & & 0.173 & -0.178 & 0.104 \\
\hline BTC & $0.244^{\star *}$ & 0.471 & 0.055 & 0.043 & 0.034 & 0.056 & 0.033 & 0.126 & -0.076 & & -0.152 & 0.068 \\
\hline TLL & $-0.200 \mathrm{~ns}$ & 0.465 & 0.065 & 0.034 & 0.040 & 0.077 & 0.033 & 0.172 & -0.095 & 0.185 & & 0.122 \\
\hline NKC & $0.179 *$ & 0.225 & 0.045 & 0.010 & 0.017 & 0.049 & -0.008 & 0.106 & -0.062 & 0.093 & -0.136 & \\
\hline
\end{tabular}

$D E$, direct effect of variable on body weight; **, significant at $p<0.01$; *, significant at $p<0.05$; ns, not significant 
Table 6. Direct and Indirect effects of morphometric traits on body weight of female Khaki Campbell ducks

\begin{tabular}{|c|c|c|c|c|c|c|c|c|c|c|c|c|}
\hline \multirow[t]{2}{*}{ Trait } & \multirow[t]{2}{*}{$\mathrm{DE}$} & \multicolumn{11}{|c|}{ Indirect effect } \\
\hline & & $\mathrm{BDL}$ & NKL & BLL & WNL & SHL & SHW & THL & THC & BTC & TLL & NKC \\
\hline$\overline{B D L}$ & $0.543^{\star \star}$ & & 0.014 & -0.012 & 0.187 & -0.141 & -0.251 & -0.001 & 0.015 & 0.487 & 0.048 & 0.022 \\
\hline NKL & $0.020^{\text {ns }}$ & 0.494 & & -0.013 & 0.175 & -0.078 & -0.203 & -0.000 & 0.012 & 0.350 & 0.040 & 0.013 \\
\hline BLL & $-0.047^{\mathrm{ns}}$ & 0.239 & 0.005 & & 0.193 & -0.040 & -0.102 & -0.000 & 0.019 & 0.202 & 0.003 & 0.018 \\
\hline WNL & $0.297^{\star *}$ & 0.125 & 0.012 & -0.031 & & -0.088 & -0.200 & -0.000 & 0.029 & 0.392 & 0.010 & 0.028 \\
\hline SHL & $-0.201^{\star *}$ & 0.440 & 0.008 & -0.009 & 0.131 & & -0.183 & -0.000 & 0.013 & 0.440 & 0.031 & 0.023 \\
\hline SHW & $-0.282^{\text {ns }}$ & 0.462 & 0.014 & -0.017 & 0.211 & -0.131 & & -0.001 & 0.022 & 0.546 & 0.045 & 0.029 \\
\hline THL & $-0.001^{\text {ns }}$ & 0.456 & 0.010 & -0.008 & 0.039 & -0.086 & -0.166 & & -0.003 & 0.261 & 0.057 & 0.008 \\
\hline THC & $0.036^{\text {ns }}$ & 0.386 & 0.007 & -0.025 & 0.238 & -0.070 & -0.169 & 0.000 & & 0.356 & 0.002 & 0.030 \\
\hline BTC & $0.594^{\star *}$ & 0.462 & 0.012 & -0.016 & 0.196 & -0.149 & -0.259 & -0.000 & 0.022 & & 0.035 & 0.026 \\
\hline TLL & $0.077^{\mathrm{ns}}$ & 0.429 & 0.010 & -0.002 & 0.039 & -0.080 & -0.166 & -0.001 & 0.001 & 0.267 & & 0.006 \\
\hline NKC & 0.062 ns & 0.353 & 0.004 & -0.014 & 0.134 & -0.074 & -0.130 & -0.000 & 0.017 & 0.249 & 0.007 & \\
\hline
\end{tabular}

$D E$, direct effect of variable on body weight; **, significant at $p<0.01 ; n s$, not significant

Table 7. Direct and Indirect effect of morphometric traits on body weight of male Pekin ducks

\begin{tabular}{lllllllllllll}
\hline Trait & DE & \multicolumn{1}{c}{ Indirect effect } \\
\cline { 3 - 13 } & & BDL & NKL & BLL & WNL & SHL & SHW & THL & THC & BTC & TLL & NKC \\
\hline BDL & $0.508^{\text {** }}$ & & -0.018 & 0.095 & -0.072 & 0.057 & 0.027 & -0.021 & 0.109 & 0.290 & -0.033 & -0.041 \\
NKL & $-0.039^{\text {ns }}$ & 0.239 & & 0.164 & -0.068 & 0.074 & 0.034 & -0.022 & 0.132 & 0.182 & -0.036 & -0.050 \\
BLL & $0.278^{*}$ & 0.173 & -0.023 & & -0.062 & 0.064 & 0.029 & -0.019 & 0.129 & 0.111 & -0.027 & -0.037 \\
WNL & $-0.101^{\text {ns }}$ & 0.361 & -0.026 & 0.170 & & 0.067 & 0.032 & -0.021 & 0.130 & 0.216 & -0.038 & -0.038 \\
SHL & $0.096^{\text {ns }}$ & 0.300 & -0.030 & 0.186 & -0.071 & & 0.043 & -0.025 & 0.153 & 0.189 & -0.041 & -0.061 \\
SHW & $0.045^{\text {ns }}$ & 0.305 & -0.030 & 0.178 & -0.071 & 0.092 & & -0.024 & 0.148 & 0.182 & -0.041 & -0.055 \\
THL & $-0.029^{\text {ns }}$ & 0.371 & -0.030 & 0.183 & -0.074 & 0.082 & 0.037 & & 0.150 & 0.239 & -0.043 & -0.060 \\
THC & $0.163^{\text {ns }}$ & 0.340 & -0.032 & 0.220 & -0.081 & 0.090 & 0.041 & -0.027 & & 0.206 & -0.043 & -0.061 \\
BTC & $0.337^{\text {** }}$ & 0.437 & -0.021 & 0.092 & -0.065 & 0.054 & 0.024 & -0.021 & 0.099 & & -0.032 & -0.041 \\
TLL & $-0.047^{\text {ns }}$ & 0.361 & -0.030 & 0.161 & -0.081 & 0.084 & 0.039 & -0.026 & 0.150 & 0.229 & & -0.058 \\
NKC & $-0.073^{\text {ns }}$ & 0.284 & -0.027 & 0.139 & -0.053 & 0.081 & 0.034 & -0.024 & 0.135 & 0.189 & -0.038 & \\
\hline
\end{tabular}

$D E$, direct effect of variable on body weight; **, significant at $p<0.01$; *, significant at $p<0.05$; ns, not significant

Table 8. Direct and Indirect effect of morphometric traits on body weight of female Pekin ducks

\begin{tabular}{|c|c|c|c|c|c|c|c|c|c|c|c|c|}
\hline \multirow[t]{2}{*}{ Trait } & \multirow[t]{2}{*}{$\mathrm{DE}$} & \multicolumn{11}{|c|}{ Indirect effect } \\
\hline & & $\overline{B D L}$ & $\overline{N K L}$ & $\overline{B L L}$ & WNL & $\overline{\mathrm{SHL}}$ & SHW & $\overline{T H L}$ & THC & BTC & $\overline{T L L}$ & NKC \\
\hline$\overline{\mathrm{BDL}}$ & $0.683^{\star \star}$ & & 0.206 & -0.119 & 0.046 & 0.970 & -0.230 & -0.556 & -0.135 & -0.022 & 0.058 & 0.021 \\
\hline NKL & $0.226^{\mathrm{ns}}$ & 0.622 & & -0.136 & 0.088 & 1.065 & -0.257 & -0.609 & -0.162 & -0.022 & 0.058 & 0.023 \\
\hline BLL & $-0.271^{\mathrm{ns}}$ & 0.301 & 0.113 & & 0.088 & 0.886 & -0.184 & -0.311 & -0.143 & -0.014 & 0.043 & 0.020 \\
\hline WNL & $0.200 \mathrm{~ns}$ & 0.157 & 0.099 & -0.119 & & 0.599 & -0.138 & -0.338 & -0.137 & -0.003 & 0.014 & 0.013 \\
\hline SHL & $1.197^{\star}$ & 0.553 & 0.201 & -0.201 & 0.100 & & -0.262 & -0.596 & -0.173 & -0.020 & 0.061 & 0.028 \\
\hline SHW & $-0.270^{\mathrm{ns}}$ & 0.581 & 0.215 & -0.184 & 0.102 & 1.161 & & -0.602 & -0.177 & -0.022 & 0.058 & 0.027 \\
\hline THL & $-0.662^{\mathrm{ns}}$ & 0.574 & 0.208 & -0.127 & 0.102 & 1.077 & -0.246 & & -0.150 & -0.017 & 0.059 & 0.023 \\
\hline THC & $-0.190^{\mathrm{ns}}$ & 0.485 & 0.192 & -0.203 & 0.144 & 1.089 & -0.251 & -0.523 & & -0.019 & 0.048 & 0.027 \\
\hline BTC & $-0.026^{\mathrm{ns}}$ & 0.581 & 0.192 & -0.146 & 0.022 & 0.934 & -0.224 & -0.444 & -0.137 & & 0.058 & 0.022 \\
\hline TLL & $0.074^{\mathrm{ns}}$ & 0.540 & 0.179 & -0.157 & 0.038 & 0.982 & -0.213 & -0.530 & -0.124 & -0.020 & & 0.021 \\
\hline NKC & $0.032^{\mathrm{ns}}$ & 0.444 & 0.163 & -0.173 & 0.082 & 1.041 & -0.224 & -0.477 & -0.162 & -0.018 & 0.048 & \\
\hline
\end{tabular}

$D E$, direct effect of variable on body weight; **, significant at $p<0.01$; *, significant at $p<0.05$; ns, not significant 
The optimum regression models for the prediction of BWT in Khaki Campbell ducks included BDL, $\mathrm{SHL}, \mathrm{BTC}$ and NKC (males) and BDL, WNL and BTC (females); while in their Pekin counterparts, $\mathrm{BDL}, \mathrm{BLL}$ and BTC (males) and BDL and SHL (females) were incorporated (Table 9). All these variables had positive and significant direct effects on BWT. The coefficient of determination $\left(R^{2}\right)$, which is a measure of reliability of each regression model, was also very high in each case.

Table 9. Optimum regression models for the estimation of body weight from morphometric traits based on sex

\begin{tabular}{|c|c|c|}
\hline Model & SE & $\mathrm{R}^{2}$ \\
\hline \multicolumn{3}{|l|}{ Khaki Campbell } \\
\hline BTW $=-0.538+0.024 \mathrm{BDL}+0.049 \mathrm{SHL}+0.017 \mathrm{BTC}+0.023 \mathrm{NKC}$ & 0.03 & 0.9 \\
\hline of $\mathrm{BTW}=-0.144+0.019 \mathrm{BDL}+0.012 \mathrm{WNL}+0.016 \mathrm{BTC}$ & 0.02 & 0.9 \\
\hline \multicolumn{3}{|l|}{ Pekin } \\
\hline O BTW $=-0.344+0.018 \mathrm{BDL}+0.070 \mathrm{BLL}+0.022 \mathrm{BTC}$ & 0.02 & 0.9 \\
\hline o $\mathrm{BTW}=0.001+0.026 \mathrm{BDL}+0.070 \mathrm{SHL}$ & 0.04 & 0.9 \\
\hline
\end{tabular}

O. male; , female; SE, standard error; $R^{2}$, coefficient of determination

\section{Discussion}

The results of this study revealed that there was a genetic variability between the two breeds of exotic ducks. Individual genetic variation within population is the figure of the heritable genetic expression variation. Genetic expression is the characteristic possessed by an individual as distinctive morphological feature (Purwantini et al. 2013). The current values on BWT are lower than the range 3124-3051g reported for seven weeks old Pekin ducks in Poland (Kokoszyński and Bernacki 2011). This may however be attributed to the improved strains used in the latter as well as environmental effect. The higher values obtained for Pekin duck in this study reflect its meat attributes while Khaki Campbell is known for egg production. These exotic ducks can be improved by exploiting the principle of phenotypic plasticity (Auld et al. 2010) to obtain better strains (Kokoszyński and Bernacki 2010) to complement the local Muscovy ducks and can also be used to upgrade some other indigenous ducks for better performance in a systematic breeding program. Padhi (2010) reported that crossbreds perform well in respect to different traits than the purebred ducks and may be used to take advantage of heterosis. The present findings are consistent with the report of Gois et al. (2012) where male body weight and body measurements were found to be higher than those of females showing dimorphism in the domestic duck of Brazil. Similar findings had been reported by earlier workers in ducks (Teguia et al. 2008; Yakubu 2011) and other poultry species (Ajayi et al. 2012), suggesting that sexual dimorphism in ducks is manifested with respect to a large number of body attributes and in most breeds. This may be attributed to sex hormones which may promote larger muscle development in males than in females (Guni et al. 2013). According to (11), sexual size dimorphism is under a strong influence of genetic and environmental factors and shaped by natural and sexual selection. The degree and direction of sexual size dimorphism often is a consequence of different selective regimes acting separately on males and females. Knowledge on sexual dimorphism can provide important basic information for selection and breeding programs (Mccracken et al. 2000).

The positive and significant phenotypic correlation coefficients between body weight and other body measurements imply that these body measurements are concomitantly linked to body weight. This is an indication that these easily measured parts can be used as criteria for estimation of body weight, and hence can also be used as selection criteria in order to improve body weight (Malomane et al. 2013).

Though correlation analysis measures the relationship between dependent and independent characters, it does not provide exact picture of how much a character constitute on its own and via other characters as the dependent trait. According to Lorentz et al. (2011), there is a limitation in the evaluation of correlation coefficients as they only indicate the magnitude and the direction of a linear association between the traits without revealing the cause-effect relationships. In a related study, Malomane et al. (2013) reported that it is difficult to directly predict body weight from highly correlated morphological traits through multiple regressions. Under such circumstances, path coefficient 
analysis is helpful in the resolution of correlation into components of direct and indirect effects (Marjanovic-Jeromela et al. 2007). The first component is the path coefficient (beta weight) that measures the direct effect of the predictor variable on the response variable. The second component estimates the indirect effect of the predictor variable on the response variable through other predictor variables. Path analysis can contribute to the understanding of the composition of traits of economic interest, indicating the most useful traits for accomplishing pre-selection. This was buttressed by Ren et al. (2008) that path analysis can be used to predict meat production of mature Shaoxing duck and applied for indirect breeding of new duck line from the population of worse reproduction performance duck. To increase the meat yield and egg production of ducks requires the genetic improvement of body weight. In this wise, BDL, BTC, SHL, BLL and NKC appeared promising in the present study. However, BDL appeared to be the trait of utmost importance considering its inclusion in all the four optimum regression models.

\section{Conclusion}

The study revealed that Pekins had superior advantage over Khaki Campbells in all the body traits investigated. Sexual dimorphism in all the traits was also in favor of males. Path analysis technique explored the interdependence in the original eleven morphometric traits and helped to summarize and explain the correlation patterns among these traits more objectively than is possible by conventional correlations. Although BDL was the trait most associated with BWT in this study, other variables such as BTC, SHL, BLL and NKC appeared promising based on genotype and sex. These morphometric traits could be used in breeding programs as a measure of direct selection for ducks with better BWT traits, although this should be consolidated with a genetic study to provide information about the heritability and genetic correlation of these variables with BWT.

\section{Acknowledgement}

The authors are grateful to the staff of National Veterinary Research Institute, Vom-Jos, Plateau State, Nigeria for their assistance during data collection.

\section{References}

Adzitey F, Adzitey SP (2011). Duck production: Has a potential to reduce Poverty among Rural Households in Asian Communities - A Review. Journal of World's Poultry Research, 1: 7-10.

Ajayi OO, Yakubu A, Jayeola OO, Imumorin IG, Takeet MI, Ozoje MO, Ikeobi CON, Peters SO (2012). Multivariate analysis of sexual size dimorphism in domestic turkeys (Meleagrisgallopavo) in Nigeria. Tropical Animal Health and Production, 44: 10891095.

Auld JR, Agrawal AA, Relyea RA (2010). Reevaluating the costs and limits of adaptive phenotypic plasticity. Proceedings of the Royal Society B, 277: 503-511.

Eyduran E, Topal M, Sonmez AY (2010). Use of factor scores in multiple regression analysis for estimation of body weight by several body measurements in brown trouts (Salmotruttafario). International J ournal of Agriculture and Biology, 12: 611-615.

Gois FD, Almeida ECJ, Farias FRV, Silva FOL (2012). Preliminary study on sexual dimorphism Duck Grey's Catolé (Cairinamoschata). Actas I beroamerican as de Conservation Animal, 2: 95-98.

Guni FS, Katule AM, Mwakilembe PAA (2013). Characterization of local chickens in selected districts of the Southern Highlands of Tanzania: II. Production and Morphometric traits. Livestock Research for Rural Development, 25.

Huang JF, Pingel H, Guy G, Lukaszewicz E, Baeza E, Wang SD (2012). A century of progress in waterfowl production, and a history of the WPSA Waterfowl Working Group. World's Poultry Science Journal, 68: 551563.

Kokoszyński D, Bernacki Z (2011). Comparison of meat performance of Pekin ducks from two conservative flocks. Journal of Central European Agriculture, 12: 215-225. 
Kokoszyński D, Bernacki Z (2010). Comparison of some meat traits in ducks from two conservative flocks. Arch. fuer Tierz. Dummerst, 53: 484-493.

Labus N, Cvijanović M, Vukov T (2013). Sexual size and shape dimorphism in Salamandrasalamandra (Amphibia, Caudata, Salamandridae) from the Central Balkans Archiv Biologica Science Belgrade, 65: 969-975.

Lorentz LH, De Genova Gaya L, Lunedo R, Ferraz] BS, De Rezende FM, Filho TM (2011). Production and body composition traits of broilers in relation to breast weight evaluated by path analysis. Sci. Agric. (Piracicaba, Brazil), 68: 320-325.

Malomane DK, Norris D, Banga CB, Ngambi JW (2013). Use of factor scores for predicting body weight from linear body measurements in three South African indigenous chicken breeds. Tropical Animal Health and Production (in press): DOI 10.1007/s11250-013-0492-2.

Marjanovic-Jeromela A, Marinkovic R, Mijic A, Zdunic Z, Ivanovskaj ankulovska M (2007). Correlation and path analysis of quantitative traits in winter rapeseed (BrasssicanapusL). Agriculture Conspectus Science, 73: 13-18.

Mccracken KV, Paton DC, Afton AD (2000). Sexual size dimorphism of the Musk duck. Wilson Bulletin, 112: 457- 466.

Mendes M, Karabayir A, Pala A (2005). Path analysis of the relationship between various body measures and live weight of American Bronze turkeys under three different lighting programs. Tar. Bil. Der, 11, 184-188.

Mir FA, Mir JI, Chandra S (2014). Detection of morphometric differentiation in Sattarsnowtrout, Schizothoraxcurvifrons (Cypriniformes: Cyprinidae) from Kashmir
Himalaya using a truss network system. Revista de Biologia Tropical, 62, 119-127.

Neto RVR, De Freitas RTF, Serafini MA, Costa AC, Freato TA, Rosa PV, Allaman IB (2012). Interrelationships between morphometric variables and rounded fish body yields evaluated by path analysis. Revista Brasileira de Zootecnia, 41: 1576-1582.

Padhi MK (2010). Production benefits of the crossbreeding of indigenous and nonindigenous ducks-growing and laying period body weight and production performance. Tropical Animal Health and Production 42: 1395-1403.

Purwantini D, Yuwanta T, Hartatik T, Ismoyowati (2013). Morphology and genetic diversity of mitochondrial DNA D-LOOP region using PCR-RFLP analysis in Magelang duck and other native duck. Journal of Indonesian Tropical Animal Agriculture, 38: 1-9.

Ren J, Liu X, Shen J, Tao Z, Jin L, Yin Z, Lu G, Tian Y, Wang D, YuanA, LiG, Yuan Q, Sun J, Zhu X, Zhu Z, Lu L (2008). Path coefficient analysis on the effect factor of meat production performance of Shaoxing Duck. China Poultry, 13:

SPSS (2010). Statistical package for the Social Sciences, (SPSS Inc., New York).

Teguia A, Ngandjou HM, Defang H, Tchoumboue J (2008). Study of the live body weight and body characteristics of the African Muscovy Duck (Cairinamoschata). Tropical Animal Health and Production, 40: 5-10.

Yakubu A (2013). Characterization of the local Muscovy duck genetic resource of Nigeria and its potential for egg and meat production. World's Poultry Science Journal 69: 931-938.

Yakubu A (2011). Discriminant analysis of sexual dimorphism in morphological traits of African Muscovy ducks (Cairinamoschata). Archivos de Zootecnia, 60: 1115-1123. 